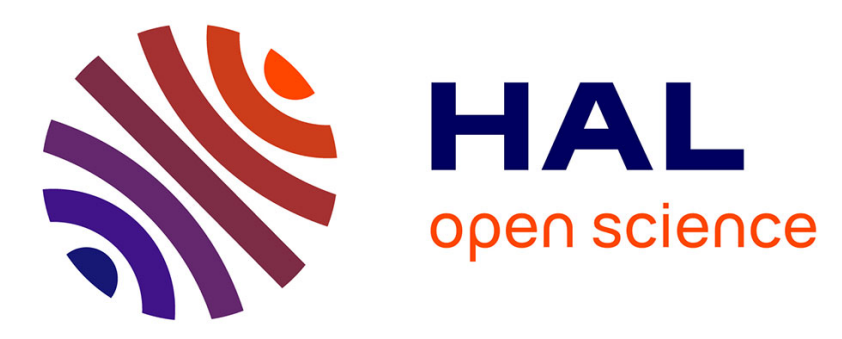

\title{
Secure and Scalable Cloud-based Architecture for e-Health Wireless Sensor Networks
}

Ahmed Lounis, Abdelkrim Hadjidj, Abdelmadjid Bouabdallah, Yacine Challal

\section{To cite this version:}

Ahmed Lounis, Abdelkrim Hadjidj, Abdelmadjid Bouabdallah, Yacine Challal. Secure and Scalable Cloud-based Architecture for e-Health Wireless Sensor Networks. International Conference on Computer Communication Networks (ICCCN), Jul 2012, Munich, Germany. pp.1. hal-00695956

\section{HAL Id: hal-00695956 https://hal.science/hal-00695956}

Submitted on 10 May 2012

HAL is a multi-disciplinary open access archive for the deposit and dissemination of scientific research documents, whether they are published or not. The documents may come from teaching and research institutions in France or abroad, or from public or private research centers.
L'archive ouverte pluridisciplinaire HAL, est destinée au dépôt et à la diffusion de documents scientifiques de niveau recherche, publiés ou non, émanant des établissements d'enseignement et de recherche français ou étrangers, des laboratoires publics ou privés. 


\title{
Secure and Scalable Cloud-based Architecture for e-Health Wireless sensor networks
}

\author{
Ahmed Lounis, Abdelkrim Hadjidj, Abdelmadjid Bouabdallah and Yacine Challal \\ \{lounisah,ahadjidj,bouabdal,ychallal\}@utc.fr \\ Université de Technologie de Compiègne \\ HEUDIASYC UMR CNRS 7253 \\ BP 20529, Compiègne Cedex \\ France
}

\begin{abstract}
There has been a host of research works on wireless sensor networks for medical applications. However, the major shortcoming of these efforts is a lack of consideration of data management. Indeed, the huge amount of high sensitive data generated and collected by medical sensor networks introduces several challenges that existing architectures cannot solve. These challenges include scalability, availability and security. In this paper, we propose an innovative architecture for collecting and accessing large amount of data generated by medical sensor networks. Our architecture resolves all the aforementioned challenges and makes easy information sharing between healthcare professionals. Furthermore, we propose an effective and flexible security mechanism that guarantees confidentiality, integrity as well as fine grained access control to outsourced medical data. This mechanism combines several cryptographic schemes to achieve high flexibility and performance.

Keywords: wireless sensor networks, healthcare, cloud computing, attribute based encryption.
\end{abstract}

\section{INTRODUCTION}

Recent advances in medical sensors, wireless technologies and Micro-Electro-Mechanical systems have enabled the development of senor nodes capable of sensing, processing and communicating several physiological signs. These lightweight miniaturized nodes collaborate to form a wireless sensor network (WSN) that simplify the supervision of patients' health. The major breakthrough of this technology is providing continuous remote patient supervision both in and out of hospital conditions. Consequently, it reduces health cost and improves the quality of life of patients as well as the treatment efficiency.

There has been a host of research works on medical WSN for patient supervision [1]. Proposed solutions have adopted a common architecture with three main components: Body Area Networks (BAN), gateways, and remote monitoring system. The BAN is a set of sensor nodes carried by the patient to collect different health information. It sends collected data via wireless communication channel to the gateway which serves as a relay node to the monitoring system through a backbone network (ADSL, WiFi, 3G or satellite). The remote monitoring system, usually a server hosted by the healthcare provider, is the heart of the architecture at which the collected data is stored, processed and accessed.

Scalability is a challenge that WSNs for medical applications should tackle. Indeed, the sampling of medical sensors is performed at high frequency which increases the amount of collected data. In addition, the frequency of sensor sampling is often increased if the condition of patients being monitored gets worse. The important size and heterogeneity of data drives a need for an increasing storage and processing capacities. Besides scalability issues, medical data could be life saving and must be accessible at any time and from everywhere. Existing solutions rely on a centralized paradigm to store and process sensed data thus cannot tackle the aforementioned challenges. We definitely need new innovative solutions to meet the great challenges of handling the exponential growth in data generated by sensors.

Considering social, ethical and legal aspects of medical systems, data collected by sensor networks is highly sensitive and should be managed properly to guarantee patients' privacy. Therefore, it is essential to ensure security of data during transmission as well as during storage. Access to patient information must be strictly limited to authorized users in order to guarantee the confidentiality. Since data is vital for medical diagnosis, data integrity should be verified to prevent wrong treatments because of malicious or erroneous modifications. Access to medical data is often governed by complex policies that distinguish between each part of the data and each user privileges. Therefore, providing fine-grained access control that supports dynamic and complex organizational policies is a very hard challenge. Practical issues, such as security management, overhead and scalability of the access control with the number of users, also need to be considered. While lot of research works have been carried out in medical wireless sensor networks, only few studies have been achieved regarding security and existing solutions are far from mature [2].

In this paper, we address the challenge of data management in wireless sensor networks for patient supervision. We propose a secure and scalable architecture for collecting and accessing large amount of data generated by medical sensor networks. We leverage cloud computing technology to dynamically scale storage resources via on demand provisioning. Furthermore, we propose an innovative security scheme that eliminates potential security threats of medical data outsourcing and guarantees confidentiality, integrity as well as fine grained access control. Our contributions in this 
work are many folds: First, we propose a new cloud based architecture for medical wireless sensor networks. Second, we show how we guarantee the confidentiality and the integrity of outsourced medical data without involving patients or doctors interventions. Third, we propose an innovative access control which allows implementing complex and dynamic security policies necessary to medical application while reducing the management and processing overhead. More specifically, we combine Ciphertext Policy Attribute Based Encryption (CP$\mathrm{ABE}$ ) and symmetric encryption to achieve fine grained access with low computation overhead.

The rest of the paper is organized as follows. In section II we review some related works. In section III we present our proposed architecture. In section IV we review attribute based encryption basics necessary to the understanding of our proposed access control. In section $\mathrm{V}$ we describe the security services that are ensured by our architecture. In section VI, we analyze the security of our solution. In section VII we conclude the paper and shed some light on future directions.

\section{RELATED WORKS}

Scalability via on-demand resource provisioning and virtually infinite data storage capacity makes the cloud computing [3] compelling for managing data generated by WSNs. Cloud computing eases storage, processing and sharing of sensor data and provides anywhere/anytime access to supervision applications. Research works on coupling WSN and the cloud are still in their early infancy. A recent paper [4] tried to identify the opportunities and challenges of connecting wireless sensor networks to the Cloud. Also, few papers introduced cloud computing to different WSN applications such as industrial supervision [5], patient data collection [6], energy monitoring [7] and environmental monitoring [8]. However, all these papers described preliminary works and ignored the challenges induced by combining WSN and cloud computing.

Authors in [9] proposed a framework based on a publish/subscribe model which facilitates WSN-Cloud connection. In another paper [10], they used this framework to monitor human activities and to share information among doctors, caregivers, and pharmacies. However, authors did not discuss the security requirements for such a framework. In ESPAC [11], data collected from patients are sent to the hospital server before being stored on the Cloud. Despite taking advantage of the cloud to offer unlimited data storage, the scalability of this scheme is limited. Indeed, the hospital server is a bottleneck (single point of failure) that may crash in the case of flash crowd. In addition, no data storage neither data access are possible on the cloud if the hospital server is out of order or inaccessible.

The storage of sensitive data over untrusted servers requires cryptography techniques in order to keep data confidential and preserve patients' privacy. Various solutions, based on symmetric or public cryptography, have been proposed to provide cryptographic access controls that allow storage and sharing of data on untrusted servers [12][13][14][15][16]. However, These techniques do not support fine grained access control required by medical applications. Indeed, they are not scalable with the number of users and introduce high complexity in key distribution and management.

Recent works leveraged new cryptography techniques, such as Role Based Access Control (RBAC) and Attribute Based Encryption (ABE), to provide fine-grained access control required by personal medical systems. Ibraimi et al. [17] applied Ciphertext Policy ABE (CP-ABE) to enable patients to securely store and share their health record on external third party servers. Barua et al. used bilinear pairing and ABE to guarantee data confidentiality and integrity as well as user privacy and authentication in cloud-based medical systems. In [18], authors proposed a novel practical framework for fine-grained data access control to medical data in Cloud. To avoid high key management complexity and overhead, they organized the system into multiple security domains where each domain manages a subset of users. Unfortunately, all these works adopted a patient-centric approach where each patient generates his own security keys and distributes them to authorized users. We argue that the patient-centric approach is not applicable to manage data collected by WSN for patient supervision. Indeed, the access policies that govern such systems are often complex to be defined by the patient. In addition, the healthcare organization is the legal owner and the responsible for patient's health data during his hospitalization within the hospital or at home settings. Consequently, security policies must be fixed by the healthcare organization rather than the patient. Finally, the patient being monitored by the sensor network can have serious health problems which make him unable to define a security policy in this reduced-mobility state.

\section{OUR PROPOSED ARCHITECTURE}

In this section, we describe our architecture which enables a healthcare institution, such as a hospital or a clinic, to manage data collected by WSN for patient supervision. The proposed architecture is scalable and able to store the large amount of data generated by sensors. Since these data are highly sensitive, we propose a new security mechanism to guarantee data confidentiality, data integrity and fine grained access control. Unlike existing patient-centric systems, security configuration and key management in our solution are totally transparent to users (patients and doctors) and do not require their interventions.

In order to achieve the aforementioned objectives, we propose the architecture described in figure 1. This architecture considers two categories of users, healthcare professionals and patients, and is composed of the following components: (1) the WSN which collects health information from patients, (2) the monitoring applications which allow healthcare professionals to access to stored data, (3) the Healthcare Authority (HA) which specifies and enforces the security policies of the healthcare institution and (4) the cloud servers which ensure data storage. By storing data on the cloud, our architecture offers virtually infinite storage capacity and high scalability. Indeed, the architecture increases its storage capacity, through 


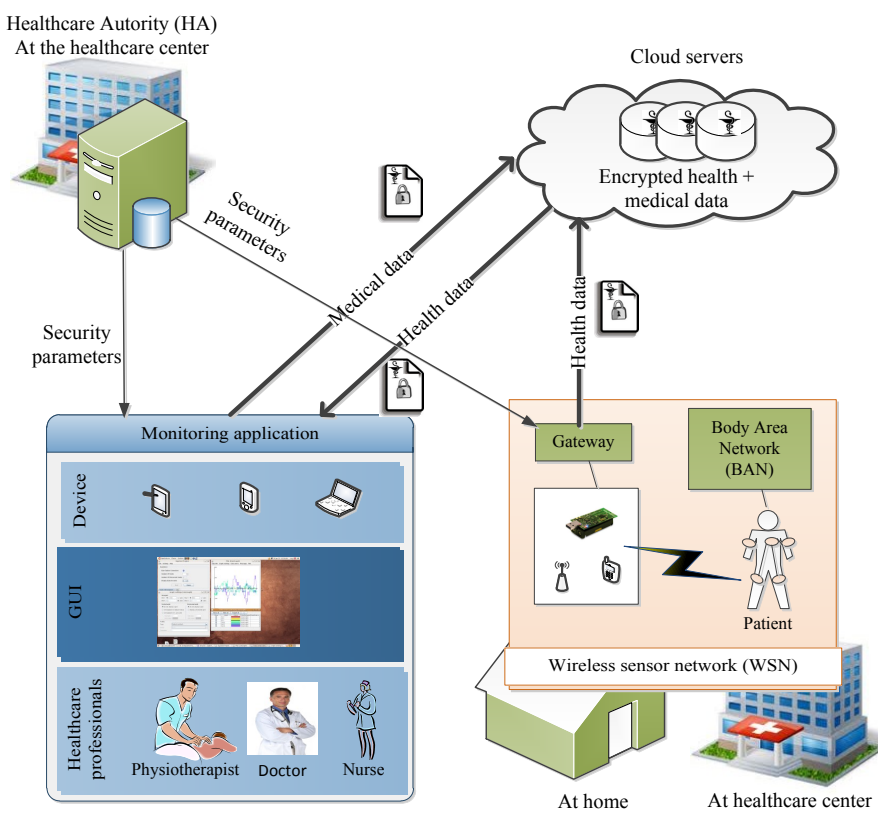

Fig. 1. The proposed architecture

on-demand provisioning feature of the cloud, whenever it is necessary. In addition, it offers enormous convenience to the healthcare institution since it does not have to care about the complexity of servers' management.

To achieve fine-grained access control, we can use attribute based encryption (ABE) to encrypt data before storing them on the cloud. However, integrating ABE into medical systems is a real challenge. In $\mathrm{ABE}$, data are encrypted with an access structure which is the logical expression of the access policy (eg: the data can be accessed by physician in cardiology division or by nurses). The cyphertext (encrypted data) can be decrypted by any user if his secret key has attributes that satisfy the access policy. The power of $\mathrm{ABE}$ is that we do not need to rely on the storage server for avoiding unauthorized data access since the access policy is embedded in the cyphertext itself. However, this characteristic becomes an inconvenient when the access policy changes. Indeed, to apply a new access policy to a file, we must download it, re-encrypt it with a new access structure and upload it again to the cloud. The second challenge faced with the integration of ABE is keys and access structures management. Indeed, the questions of who should generate the access structure that govern the security policy and who should generate and distribute keys necessary to access to the data are a real challenge in medical systems. To answer these questions, existing ABE-based systems adopted a patient centric approach that we showed unsuitable for our application.

To tackle the first challenge of ABE integration, we propose to use both symmetric cryptography and $\mathrm{ABE}$ to encrypt data. More specifically, we propose to encrypt each file with a randomly generated symmetric key (RSK) and encrypt the RSK with ABE. Both the encrypted file and the encrypted $\mathrm{RSK}$ are sent to the cloud for storage to allow fine grained data sharing with authorized users. Indeed, if a user has a secret key that satisfies the ABE access policy, he will be able to decrypt the RSK and hence to decrypt the file. Furthermore, if the file access policy changes, we should download and re-encrypt the RSK rather than the whole file. This lead to a significant gain in data communication and encryption operations. Finally, our solution has less encryption overhead compared to the naif utilization of $\mathrm{ABE}$ to encrypt the whole file. In fact, $\mathrm{ABE}$ consumes much more processing power than symmetric cryptography when we use complex access policy [19] like ones used in medical systems.

To tackle the second challenge, which is mastering the complexity of security management, we introduce an entity that we call Healthcare Authority (HA). The HA specifies and enforces the security policies of the healthcare institution. It is used by the administrators of the healthcare institutions to define rules as "who can access to what". Based on these rules, the HA generates and sends to each user his ABE security parameters which are a pair of access structure and secret key. The secret key is tagged with the user attributes set which represent the user privileges. This information is required to decrypt data that the user is allowed to access. The access structure represents the access policy that protects the user data. When a user encrypts the random symmetric key (RSK) that protects his data using this structure, he can be sure that only authorized users (who have the correct attributes) can decrypt and access to his data. Introducing the HA releases users from creating and distributing access structures and secret keys. Consequently, it improves the system usability since a patient has no action to do to secure his data. Also, the healthcare professionals transparently access to data falling under their scope. All the details of security operations are given in section $\mathrm{V}$.

In our architecture, each patient has a personal WSN composed of a set of lightweight/small sensor nodes and a gateway. A WSN enables unobtrusive and continuous health supervision of the patient at the hospital and at home settings. Sensor nodes are carried by the patient to collect different health data such as heart beats, motion and physiological signals. Each sensor node sends the collected information via a wireless communication channel to the gateway. The gateway aggregates the different health data into a file and encrypts it using the RSK. Thereafter, it sends the encrypted file along with the RSK encrypted using the access structure obtained from the HA to the cloud.

The monitoring application allows healthcare professionals to supervise their patients and enables them to access to a patient's data anytime and from everywhere using a computer or a Smartphone. The monitoring application downloads the required data from the cloud and decrypts it using its secret key. In addition, it allows the healthcare professionals to add medical data, such as reports, diagnostics and prescriptions, to the patient's information. The medical data is also encrypted and stored on the cloud along with the patient's health data. Similarly to the gateway, the monitoring application encrypts the medical data using a RSK and the access structure obtained 
from the HA.

\section{BACKGROUNDS: ATTRIBUTE-BASED ENCRYPTION}

Attribute-based encryption (ABE) is a recent promising cryptographic method proposed by Sahai and Waters in 2005 [20]. The ABE technique extends the identity-based encryption to enable expressive access policies and fine-grained access to encrypted data. In ABE, the access control decision is based on a set of attributes and the concept of access structure described as follows :

- Universal attributes set $(\mathbf{U})$ : is the set of all attributes that describe data properties, user properties and environment properties.

- Access structure: is an access policy that designs who can access to what. It is built from an access tree (T) which can be seen as a logical expression combining several attributes through AND, OR or other operators (figure 2). Each non-leaf node of the tree represents a threshold gate, described by its children and the threshold gate value (AND, OR or other operators). Each leaf node of the tree is described by an attribute from $U$ and a value.

In figure 2, we give an example of an access tree which is derived from the following logical expression: ((speciality=physician AND (division=cardiology OR cardiology=pulmonary) OR (cardiology=gerontology AND (speciality=nurse OR speciality=physician))). This expression means that data can be accessed by all physicians working in cardiology, pulmonary or gerontology divisions, as well as all nurses working in gerontology division have access.

Key-Policy Attribute-Based Encryption (KP-ABE) [21] and Ciphertext-Policy Attribute-Based Encryption (CP-ABE) [19] are the two main variants of ABE. KP-ABE assigns to each file a set of attributes to be encrypted, and assigns to each user an access structure, that represents his access scope, for data decryption. On the contrary, $\mathrm{CP}-\mathrm{ABE}$ assigns to each file an access structure to be encrypted, and uses a set of attributes to generate the user's key for data decryption. In medical systems, healthcare professionals are assigned particular roles (eg. general practitioner, nurse), and through those role they get permissions to access to particular data. Implementing these policies is easier and more efficient using $\mathrm{CP}-\mathrm{ABE}$ than using KP-ABE. Indeed, we can describe the role of each healthcare professional by assigning a combination of attributes. At the same time, we encrypt each file by an access structure that express the access policy. In what follows we present the basics of $\mathrm{CP}-\mathrm{ABE}$ necessary for the understanding of our architecture. More extensive description of $\mathrm{CP}-\mathrm{ABE}$ is available in [19].

A CP-ABE scheme consists of four fundamental algorithms: setup, encrypt, key generation, and decrypt.

Setup: defines the universal attributes set (U) and computes the public key (PK) and the master key (MK). The public key (PK) is used in encryption and decryption algorithms. The master key (MK) is needed to generate secret keys in the Key generation algorithm.

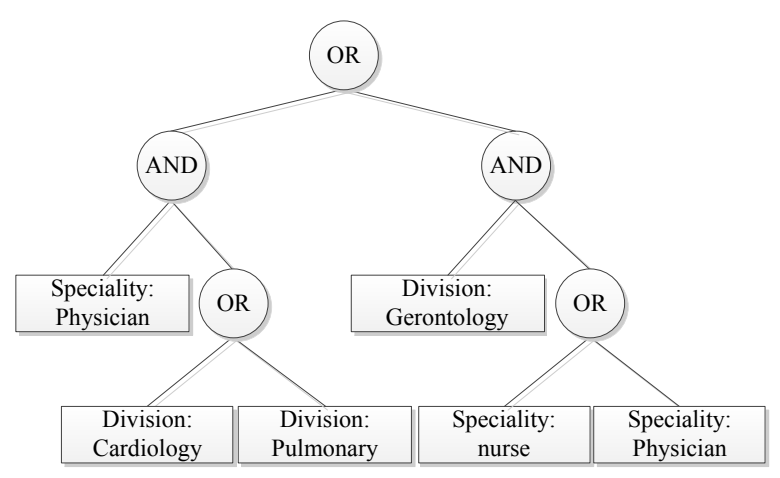

Fig. 2. An access tree T

Encryption (PK, M, A): it takes as input the public key PK, a message $\mathrm{M}$, and an access structure $\mathrm{A}$ built over the universal attributes set $(\mathrm{U})$. This algorithms encrypts the message $\mathrm{M}$ according to the access policy that is defined by the access structure A, and gives as output the ciphertext CT. Only users having a set of attributes corresponding to the access structure A can decrypt the ciphertext (CT).

Key generation (MK, S): this algorithm takes as input a master key MK and the user set of attributes $\mathrm{S}$ and generates the user's secret key SK.

Decryption (PK, CT, SK): it takes as input the public key $\mathrm{PK}$, the ciphertext CT and a secret key SK. It returns a message $\mathrm{M}$ that is plaintext of CT if the set of attributes corresponding to SK satisfies the access structure A of CT.

\section{SECURITY SERVICES IMPLEMENTATION}

In this section, we give the security model and define the services that are ensured by our architecture. Then, we details the implementation of these security services.

\section{A. Security Model}

Our system is composed of the following parties: many users (patients and healthcare professionals), many cloud servers and the healthcare authority (HA) server. We assume that communication channels between users, the HA and cloud servers are secured by a security protocol such as SSL. Even if SSL guarantees data confidentiality and integrity during transfer, we should encrypt data at the user level because we consider that cloud servers are untrusted. Indeed, data are stored on clouds operated by companies that may disclose personal information to third parties. For legal and ethical concerns, the cloud provider should neither be able to access to patients' data nor perform data mining or patients profiling. Furthermore, we consider that cloud servers might collude with some malicious or revoked users for illegal data access. Similarly, users might collude together to illegitimately access to file contents. The Healthcare Authority (HA) assures keys and access policies management. We consider that the HA is trusted and secured since it is hosted by the healthcare institution. Finally, we assume that each party has a public/private key pair and the public key can be easily obtained by other parties. 


\section{B. Security services}

Our architecture guarantees the following security services.

Fine-grained Access control: Our solution ensures healthcare information confidentiality and scalable fine-grained access control to data stored on the cloud. Furthermore, it ensures access control in multi-writers access mode on medical data.

Integrity and authenticity: Our solution ensures message integrity during transfer between two parties. Furthermore, it ensures data integrity during storage on cloud servers. Also, each party authenticates the origin of each message received from others parties.

Availability: Our solution ensures availability of service for legitimate users when they need it, and it is resilient when a large groups of legitimate users' access at the same time (flash crowd). Furthermore, it is resilient to Denial of Service attacks.

Collusion resistance: Our solution enforces the access control system and guarantees that users (patients/healthcare staffs) cannot collude together to get illegitimate access to medical data. Our architecture resists against the collusion attacks to avoid any unauthorized access to medical data.

\section{C. security implementation}

1) System initialization: At the installation of our architecture, the HA creates the universal attributes set and calls the $\mathrm{ABE}$ setup algorithm to generate the master key $(\mathrm{Mk})$ and the public key (Pk). The Mk must remain secret while the Pk must be shared with all users since they need it to decrypt data. To share the Pk, the HA signs it with its private key and sends it, along with the signature, to cloud servers. Once the Pk on the cloud, users can download it and check its authenticity thanks to the signature.

2) Adding new user: When a new patient is admitted to the hospital, the Healthcare Authority gives him a secret key and an access structure. The access structure allows him to encrypt his data before uploading it on cloud servers and ensures that only authorized users can access to it. The secret key allows him to access to medical data on which he has right. The following steps are performed each times a new patient $P$ joins the system :

1) The HA generates a couple of private/public keys $\left(\operatorname{Priv}_{P}, P u b_{P}\right)$ for the patient $P$.

2) The HA calls the key generation algorithm of CP$\mathrm{ABE}$ to generate the secret key $S K_{P}$. Furthermore, it builds the access structure $A R_{P}$ that the patient $P$ will use to encrypt his health data.

3) The HA asks the cloud to add the patient $P$ to the users lists.

4) Upon receiving the patient addition request, the cloud adds the patient $\mathrm{P}$ and his public key $P u b_{P}$ to the users list $(L U)$.

5) When the patient's gateway establishes a connection to the HA for the first time, it receives the corresponding secret key $S K_{P}$, access structure $A R_{P}$ and private key $\operatorname{Priv}_{P}$.
The difference between the security parameters of a patient and a healthcare professional comes from the fact that a patient needs to encrypt health data which can be only readded while a healthcare professional needs to encrypt medical data which can be both readded and modified. The read access policy and the write access policy which govern a medical data may be different. For example, a nurse can only read a report while a doctor can read and modify it to add comments. Consequently, the healthcare professional should obtain two access structures for read and for write policies. The following steps are performed each times a new healthcare professional $H P$ joins the system :

1) The HA generates a couple of private/public keys $\left(\right.$ Priv $\left._{H P}, P u b_{H P}\right)$ for the $H P$.

2) The HA calls the key generation algorithm of CP$\mathrm{ABE}$ to generate the secret key $S K_{H P}$. Furthermore, it builds an access structure $A R_{H P}$ that the $H P$ will use to encrypt the medical data. Also, it builds another access structure $A W_{H P}$ for protecting the write mode. We will explain how the $A W_{H P}$ is used in the medical data management subsection.

3) The HA asks the cloud to add the $H P$ to the users list.

4) Upon receiving the $H P$ addition request, the cloud adds the $H P$ and his public key $P u b_{H P}$ to the users list (LU).

5) When the $H P$ establishes a connection to the HA for the first time, its application receives the corresponding secret key $S K_{H P}$, private key $\operatorname{Priv}_{H P}$ and access structures $A R_{H P}, A W_{H P}$.

3) health data management: Health data files are information collected by the WSN and can be accessed only in reading mode. The gateway continuously receives information collected by sensor nodes and executes the following algorithm when this data is ready to be uploaded to the cloud:

1) Assigns a unique identifier $I D$ to the health data file $F$

2) Generates a random secret key $R S K$ for a symmetric cryptography algorithm

3) Computes $H$ the hash value of the file $F$

4) Uses $R S K$ to encrypt the concatenation of the file $F$ and the hash value $H$

5) Encrypts $R S K$ with $\mathrm{CP}-\mathrm{ABE}$ encryption algorithm according to the access structure $A R_{P}$

6) Sends to the cloud the following data :

$$
\begin{array}{|l|l|l|}
\hline I d & \{R S k\}_{A R_{P}} & \{(\text { Data }+H)\}_{R S K} \\
\hline
\end{array}
$$

Once stored on the cloud, the health data can be used by healthcare professionals to remotely supervise the patient or by the patient itself. When a user $U$ wants to access a health data file, he starts by downloading this file from the cloud server. After, he decrypts the $R S K$ field of the file using ABE and his secret key $S k_{U}$. If he has the right to access to this 
file (his secret key corresponds to the access structure of the patient $P$ ), he gets the correct $R S K$ and hence decrypts the file. After the decryption, the user checks the integrity of the content thanks to the hash value. If he detects that the data file was altered he signals it to the Healthcare Authority. Figure 3 shows the different steps performed from adding a new patient until its supervision.

4) Medical data management: The medical data (such as reports, diagnostics and prescriptions) are created by healthcare professionals and can be modified by other authorized users. The read access to medical data is similar to health data. However, to control medical files updates, we assign to each file a password that only the cloud and users authorized to modify the file can find. To let a user upload a new version of a file $F$, the cloud asks him for the file password. If the user provides the correct password, the new file version is accepted. When a healthcare professional $H P$ creates a new medical file $F$, he performs the following actions:

1) Assigns a unique identifier $I D$ to the medical data file $F$

2) Generates a random secret key $R S K$ for a symmetric cryptography algorithm

3) Generates a random password $P A S S$ for protecting controlling the write access

4) Computes $H$ the hash value of the file $F$

5) Uses $R S K$ to encrypt the concatenation of the file $F$ and the hash value $H$

6) Encrypts $R S K$ with CP-ABE encryption algorithm according to the read access structure $A R_{H P}$

7) Encrypts $P A S S$ with CP-ABE encryption algorithm according to the write access structure $A W_{H P}$

8) Encrypts $P A S S$ with the public key of the cloud

9) Sends to the cloud the following data :

\begin{tabular}{|c|l|l|l|}
\hline$I d$ & $\{R S k\}_{A R_{H P}}$ & $\{P A S S\}_{A W_{H P}}$ & $\{P A S S\}_{P_{\text {uub }} \text { Cloud }}$ \\
\hline $\multicolumn{4}{|c|}{(\text { Data }+H)}_{R S K}$ \\
\hline
\end{tabular}

To read the content of a medical file, a user $U$ performs the same actions described in the last section (access to health file). However, to modify a medical file he performs the following actions:

1) Downloads the medical file

2) Updates the file content and computes the new hash value of the file;

3) Encrypt the medical content along with the new hash value using $R S K$;

4) Decrypt the password with $\mathrm{ABE}$ and $S k_{U}$

5) Sends to the cloud an update request containing the new file along with computed password

6) Upon receiving the update request, the cloud the password of the original file using his private key Priv $_{\text {cloud }}$. The new version of the file is accepted if and only if the password computed by the cloud is equal to the password in the update request.
5) Data health deletion: This operation can be performed only by the file owner. To delete a file, the owner signs and sends a delete request to the cloud. Upon receiving this request, the cloud checks if the sender is the real owner of the file based on the signature and proceeds to the deletion.

6) Revocation: There are two types of revocation. The first one consists of limiting access to data through modifying the access policy. To change a data access policy, we should create a new data access structure and re-encrypt the desired data. The second one consists of revocation of attributes that are associated to user to limit his access scope. This operation induces more computing overhead. To allow an efficient revocation and solve this challenge, we add an expiration time attribute to each users key [19]. This expiration time represents until when the key is considered valid. Another method to deal with the revocation problem is using the proxy re-encryption technique [22] to delegate most of laborious revocation tasks to cloud servers in secure manner.

\section{SECURITY AND PERFORMANCE ANALYSIS}

\section{A. Security analysis}

Our solution guarantees message integrity, authenticity and confidentiality during data transfer through SSL protocol. Furthermore, it ensures a secure and fine grained access control to data files stored on the cloud. Indeed, data files are encrypted by a randomly generated symmetric key, and this key is encrypted by CP-ABE. The CP-ABE scheme has been proved secure in [19]. Especially, The CP-ABE scheme has been proved resistant against collusion attacks and ensuring that encrypted data cannot be accessed by unauthorized users. From this, we deduce that the random symmetric key is confidential and can be accessed only by authorized users. Consequently, the data confidentiality is guaranteed by the standard symmetric encryption security.

Since CP-ABE enables scalable and fine-grained access control, the HA is able to define and enforce expressive and personalized access structure for each user. These access structures enable us to select with fine granularity which users can access to the symmetric key of a given file. Since accessing to the symmetric key is necessary to access to the file, we deduce that these access structures enables us to select with fine granularity which user can access to a file contents. Finally, be using separate access structures for the read and the write policies, we separate between read and write access to medical data.

\section{B. Performance analysis}

$\mathrm{CP}-\mathrm{ABE}$ enables fine grained access control to data but induces important processing overhead with complex access policies like ones used in medical systems. The encryption time of CPA-ABE is linear with the number of leaf nodes of the used access structure. However, measuring the decryption time is more difficult since it significantly depends on the access tree used (number of leaf nodes, the type of used operators, the depth of the tree ...) and the set of attributes 


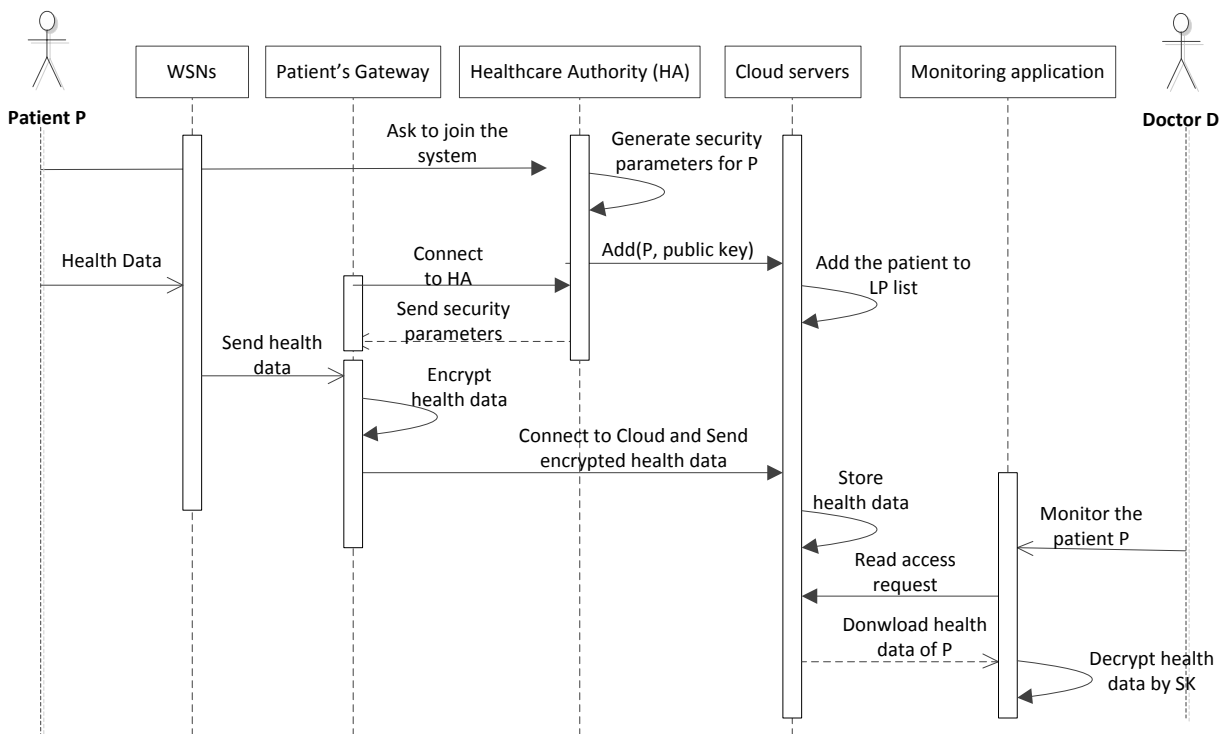

Fig. 3. Example of patient supervision

TABLE I

COMPARISON OF THE PERFORMANCE OF ABE AND OUR SOLUTION.

\begin{tabular}{clllll}
\hline Algorithm & Operation & AS1 & AS2 & AS3 & AS4 \\
\hline \multirow{2}{*}{ ABE } & Encryption (s) & 2,87 & 2,21 & 2,55 & 3,86 \\
\cline { 2 - 6 } & Decryption (s) & 1,60 & 1,70 & 1,79 & 2,80 \\
\hline \multirow{2}{*}{ Our solution } & Encryption (s) & 1,60 & 1,85 & 1,88 & 2,16 \\
\cline { 2 - 6 } & Decryption (s) & 1,13 & 1,01 & 1,05 & 1,50 \\
\hline
\end{tabular}

involved [19]. Here we present preliminary performance evaluation to show the benefit of our solution compared to ABE. We considered several random access structures and attribute sets that we can meet in a real medical system. We used the toolkit developed in [23] for ABE and the AES implementation of OpenSSL for the symmetric encryption. Table I shows a sample of encryption and decryption time obtained using ABE and our solution respectively. We plan to do more complete and thorough performance evaluations in future works.

Table I shows that ABE consumes more time than our solution both in encryption and decryption. These results match our expectations and show that our control access scheme is more efficient in terms of cryptographic operations. Indeed, our solution uses AES to encrypt the data file and uses CP-ABE to only encrypt the AES key (256 bits). Since AES is faster than ABE, we reduce the whole encryption and decryption time. This reduction vary between $27 \%$ and $47 \%$ in the studied samples. Notice that these performance evaluation do not consider the significant gain that we can achieve in revocation thanks to our access control.

\section{CONCLUSION}

In this paper, we addressed the challenge of data management in wireless sensor networks for patient supervision. We proposed a secure and scalable architecture that leverages cloud computing technology to dynamically scale storage resources via on demand provisioning. Furthermore, we proposed an innovative security scheme that eliminates potential security threats of medical data outsourcing and guarantees confidentiality, integrity without involving patients or doctors interventions. To implement complex and dynamic security policies necessary to medical application, we developed a fine grained access control that combines attributes based encryption and symmetric cryptography. This combination reduced the management overhead and the encryption/decryption time as showed by our preliminary performance evaluation. In future works, we plan to use distributed attribute-based encryption to have multi healthcare authorities. Also, we plan to perform more thorough and complete performance evaluation during encryption, decryption and revocation.

\section{REFERENCES}

[1] H. Alemdar and C. Ersoy, "Wireless sensor networks for healthcare: A survey," Computer Networks, vol. 54, no. 15, pp. 2688-2710, Oct. 2010.

[2] M. Li, W. Lou, and K. Ren, "Data security and privacy in wireless body area networks," IEEE Wireless Communications, vol. 17, no. 1, pp. 51-58, Feb. 2010.

[3] R. Buyya, C. S. Yeo, S. Venugopal, J. Broberg, and I. Brandic, "Cloud computing and emerging IT platforms: Vision, hype, and reality for delivering computing as the 5th utility," Journal of Future Generation Computer Systems, vol. 25, no. 6, pp. 599-616, Jun. 2009.

[4] R. Liu and I. J. Wassell, "Opportunities and challenges of wireless sensor networks using cloud services," in Proceedings of the workshop on Internet of Things and Service Platforms, IoTSP '11, New York, NY, USA, 2011, pp. 41-47.

[5] V. Rajesh, J. M. Gnanasekar, R. S. Ponmagal, and P. Anbalagan, "Integration of wireless sensor network with cloud," in International Conference on Recent Trends in Information, Telecommunication and Computing, ITC'10, Kochi, India, Mar. 2010, pp. 321-323.

[6] C. O. Rolim, F. L. Koch, C. B. Westphall, J. Werner, A. Fracalossi, and G. S. Salvador, "A cloud computing solution for patient's data collection in health care institutions," in Second International Conference on eHealth, Telemedicine, and Social Medicine, ETELEMED '10, St. Maarten, Netherlands Antilles, Feb. 2010, pp. 95-99. 
[7] W. Kurschl and W. Beer, "Combining cloud computing and wireless sensor networks," in Proceedings of the 11th International Conference on Information Integration and Web-based Applications \& Services, iiWAS '09, New York, NY, USA, 2009, pp. 512-518.

[8] K. Lee, D. Murray, D. Hughes, and W. Joosen, "Extending sensor networks into the cloud using amazon web services," in IEEE International Conference on Networked Embedded Systems for Enterprise Applications, NESEA'10, Suzhou, China, Nov. 2010, pp. 1-7.

[9] M. M. Hassan, B. Song, and E. Huh, "A framework of sensor-cloud integration opportunities and challenges," in Proceedings of the 3rd International Conference on Ubiquitous Information Management and Communication, ICUIMC '09, New York, NY, USA, 2009, pp. 618-626.

[10] A. M. Khattak, L. T. Vinh, D. V. Hung, P. T. H. Truc, L. X. Hung, D. Guan, Z. Pervez, M. Han, S. Lee, and Y. Lee, "Context-aware human activity recognition and decision making," in 12th IEEE International Conference on e-Health Networking Applications and Services, Healthcom'10, Lyon, France, Jul. 2010, pp. 112-118.

[11] M. Barua, X. Liang, R. Lu, and X. Shen, "ESPAC: enabling security and patient-centric access control for eHealth in cloud computing," International Journal of Security and Networks, vol. 6, no. 2/3, pp. 67-76, Nov. 2011.

[12] M. Kallahalla, E. Riedel, R. Swaminathan, Q. Wang, and K. Fu, "Plutus: Scalable secure file sharing on untrusted storage," in Proceedings of the 2nd USENIX Conference on File and Storage Technologies. Berkeley, CA, USA: USENIX Association, 2003, pp. 29-42.

[13] E.-j. Goh, H. Shacham, N. Modadugu, and D. Boneh, "Sirius: Securing remote untrusted storage," Network and distributed systems security, NDSS'03, pp. 131-145, 2003.

[14] J. Benaloh, M. Chase, E. Horvitz, and K. Lauter, "Patient controlled encryption: ensuring privacy of electronic medical records," in Proceedings of the 2009 ACM workshop on Cloud computing security, CCSW '09, New York, NY, USA, 2009, pp. 103-114.

[15] S. D. C. di Vimercati, S. Foresti, S. Jajodia, S. Paraboschi, and P. Samarati, "Over-encryption: management of access control evolution on outsourced data," in Proceedings of the 33rd international conference on Very large data bases, ser. VLDB '07, 2007, pp. 123-134.

[16] W. Wang, Z. Li, R. Owens, and B. Bhargava, "Secure and efficient access to outsourced data," in Proceedings of the 2009 ACM workshop on Cloud computing security, CCSW '09, New York, NY, USA, 2009, pp. 55-66.

[17] L. Ibraimi, M. Asim, and M. Petkovic, "Secure management of personal health records by applying attribute-based encryption," in 6th International Workshop on Wearable Micro and Nano Technologies for Personalized Health, pHealth'09, Oslo, Norway, Jun. 2009, pp. 71-74.

[18] O. Akan, P. Bellavista, J. Cao, F. Dressler, D. Ferrari, M. Gerla, H. Kobayashi, S. Palazzo, S. Sahni, X. S. Shen, M. Stan, J. Xiaohua, A. Zomaya, G. Coulson, M. Li, S. Yu, K. Ren, and W. Lou, "Securing personal health records in cloud computing: Patient-Centric and FineGrained data access control in multi-owner settings," in Security and Privacy in Communication Networks. Springer Berlin Heidelberg, 2010, vol. 50, pp. 89-106.

[19] J. Bethencourt, A. Sahai, and B. Waters, "Ciphertext-Policy AttributeBased encryption," in Proceedings of the IEEE Symposium on Security and Privacy, SP '07, Washington, DC, USA, 2007, pp. 321-334.

[20] A. Sahai and B. Waters, "Fuzzy Identity-Based encryption," in Lecture Notes in Computer Science, vol. 3494, 2005, pp. 457-473.

[21] V. Goyal, O. Pandey, A. Sahai, and B. Waters, "Attribute-based encryption for fine-grained access control of encrypted data," in Proceedings of the 13th ACM conference on Computer and communications security, CCS '06, New York, NY, USA, 2006, pp. 89-98.

[22] S. Yu, C. Wang, K. Ren, and W. Lou, "Attribute based data sharing with attribute revocation," in Proceedings of the 5th ACM Symposium on Information, Computer and Communications Security, ASIACCS '10, New York, NY, USA, 2010, pp. 261-270.

[23] [Online]. Available: http://acsc.cs.utexas.edu/cpabe/ 